\title{
Renewing the Future: Social Innovation Systems, Sector Shift, and Innoweave

\author{
Stephen Huddart
}

\author{
'I think what connects the challenge for business, the challenge" \\ for government and the challenge for communities now, is both \\ simple and difficult. We know our societies have to radically \\ change. We know we can't go back to where we were before. \\ Geoff Mulgan \\ Chief Executive, NESTA
}

\begin{abstract}
Against a backdrop of various "occupy" movements signifying civic dissatisfaction with the social contract, and in an era of fiscal restraint affecting governments and communities in many parts of the world, we need new and more effective ways to address complex social challenges. While continuous innovation is commonly understood to be a source of growth, productivity improvement, and competitive advantage in the technology and manufacturing sectors, the author's focus is on social innovation systems, designed to replace maladaptive institutions and obsolete policy frameworks with novel and disruptive means for improving outcomes on issues such as population health and climate change.
\end{abstract}

This article proposes a definition of such systems, and examines how system-level tools including impact investing, open data platforms, and "change labs" are fostering collaboration among the private, public, and community sectors. We argue that a key priority at this time is to make these and other tools and processes for social innovation available to community organizations and their government and business partners everywhere, in a manner that allows for continuous cycles of implementation and learning.

The author describes one such project currently being developed in Canada by Social Innovation Generation and other partners, called Innoweave. Innoweave is a technology-enabled social innovation system for sharing the tools and processes of social innovation with the community sector. The article concludes with a call for multi-sectoral participation in social innovation systems as an investment in society's adaptive capacity and future wellbeing.

\section{Introduction}

A recent European Community paper (BEPA, 2011; tinyurl.com/867tbem) defines social innovation as follows:

"Social innovations are innovations that are social in both their ends and their means. Specifically, we define social innovations as new ideas (products, services and models) that simultaneously meet social needs (more effectively than alternatives) and create new social relationships or collaborations. They are innovations that are not only good for society but also enhance society's capacity to act."
The philanthropic and charitable sector often acts as society's "Research and Development Department", testing new ideas at relatively low cost and enabling governments, communities, and the private sector to adopt those that deliver better outcomes. Typically, organizations proposing a social innovation - such as Roots of Empathy (rootsofempathy.org) or JUMP Math (jumpmathl.org) - operate on grant funding until able to prove that they offer a new and better solution to a social problem. While numerous breakthroughs can be cited, experience has taught the McConnell Family Foundation and its grantee partners that the political, 


\section{Renewing the Future: Social Innovation Systems, Sector Shift, and Innoweave}

Stephen Huddart

economic, and social context within which a problem occurs is often invested in maintaining the status quo and thus resists change.

The introduction of systems language points to the importance of context. Frances Westley and Nino Antadze (2009; tinyurl.com/bmqddrh) write that social innovations "involve institutional and social system change, [...] contribute to overall social resilience, and demand a complex interaction between agency and intent and emergent opportunity." A definition from the Social Innovation Generation's "Social Innovation Primer" (sigeneration.ca/primer.html) is also helpful:

"Social innovation is an initiative, product or process or program that profoundly changes the basic routines, resource and authority flows or beliefs of any social system (e.g., individuals, organizations, neighbourhoods, communities, whole societies). The capacity of any society to create a steady flow of social innovations, particularly those which re-engage vulnerable populations, is an important contributor to overall social and ecological resilience."

A social innovation system then, refers to institutional arrangements designed to produce that "steady flow of social innovations" mentioned in the previous paragraph, to address complex problems from multiple perspectives, using a range of means over time and at different levels of scale, so as to transform problems and their contexts into matrices for new understanding, new approaches, and potentially, the emergence of new systems.

\section{New Business Models: Shared Value and Impact Investing}

The need to increase private sector productivity in order to remain globally competitive and maintain living standards is well understood. Moreover, the day has passed when companies could focus on short-term profits while generating "externalities" in the form of pollution, ecosystem destruction, or social dislocation, for governments and future generations to clean up. As Michael Porter predicted over 20 years ago (Sustainable Prosperity, 2010; tinyurl.com/csocmgf), leading companies operate with enhanced consideration for environmental and social consequences. By "sharing values", such companies advance environmental and social goals in collaboration with communities. They also share their capacity with community sector partners, through strategic alliances, direct funding, and in the form of technology and skills transfer.
The diverse firms comprising the EXCEL Partnership (delphi.ca/services/the_excel_partnership/), for example, share an interest in sustainability. They collaborate on strategy, work cross-sectorally, and invest in leadingedge research and community programs. In another example, 21 forest companies are re-engineering their operations to follow the advanced sustainability principles of the Forest Stewardship Council (fsc.org) through the Canadian Boreal Forest Agreement (canadianborealforestagreement.com). This is a system-level bet that transforming a market in the public interest will confer competitive advantage and improve longterm viability, for an industry and the communities where it operates. Notably, the agreement came about in response to challenges posed by conservation organizations and foundations who continue to co-invest in its implementation.

Companies also face a challenging social context - including an aging workforce, a burgeoning Aboriginal population, and increased flows of skilled immigrants. Those whose recruitment practices address such issues will attract and retain stronger workforces. To cite an example in the skilled immigrant area, the Toronto Region Immigrant Employment Council (TRIEC; triec.ca) and Assisting Local Leaders with Immigrant Employment Strategies (ALLIES; alliescanada.ca) programs integrate philanthropic and community capacity with private-sector leadership and government support to create shared resources for learning and improving practice around recruitment, mentoring, and peer support.

Social Capital Partners (SCP; socialcapitalpartners.ca) exemplifies another promising approach. SCP works with businesses, community agencies, and governments to create hiring programs for people facing barriers to employment. It provides low-interest financing to entrepreneurs willing to add a social dimension to their human resource policies and it is working with some of Canada's largest employers to integrate advanced community hiring practices into their recruitment strategies.

Business leadership includes support for the emerging field of impact investing (Bugg-Levine and Emerson, 2011; tinyurl.com/c6ffuff). The recently established Centre for Impact Investing (impactinvesting.marsdd.com) at MaRS is incubating new hybrid financial instruments for achieving social and environmental objectives, and with philanthropic and private sector support, will soon launch the SVX (thesvX.org), a platform for capitalizing social ventures. 


\section{Renewing the Future: Social Innovation Systems, Sector Shift, and Innoweave}

Stephen Huddart

Building on the recommendations of the Canadian Task Force on Social Finance (socialfinance.ca/taskforce), private and public foundations are beginning to develop strategies for placing a portion of their endowments in impact investments. Quebec's Chantier de l'Economie Sociale (chantier.qc.ca) is a leader in this field and has a diverse portfolio of blended investments. Can it be long before universities, pension funds, and other institutions with large endowments begin to invest in a similar manner?

Vancity has partnered with the BC Government and the Vancouver Foundation to create Resilient Capital, a \$15 million dollar impact-investment fund. The Royal Bank of Canada (RBC; rbc.com) has announced a new \$20 million impact-investment fund (tinyurl.com/7qbkjyx) that is actively seeking investments in initiatives with clear environmental and social goals.

In comparison with the UK, where the government has launched Big Society Capital (bigsocietycapital.com), a bank with $£ 600$ million (approx. $\$ 930$ million US) to invest in social ventures, these are small steps, but as investors recognize that such investments can provide stable and attractive financial returns while advancing social and environmental goals, the market should expand quickly.

\section{Social Innovation in the Public Sector}

Governments caught in the downdrafts of fiscal restraint can find it necessary to simply offload employees and cut programs to stay afloat. However, when such measures are undertaken without regard for the larger systems within which we all must navigate, the effect can be to simply offload the problem onto another level of the system, usually a subsidiary one, at the bottom of which stand households and families, or to hand the problem to the future in the form of growing social and environmental deficits, as we currently do with $\mathrm{Ab}$ original youth and climate change.

Investments in social innovation allow systems and communities to adapt during times of austerity, as Geoff Mulgan (2012; tinyurl.com/77jn5dn) recently explained in Toronto. Just as the private sector has learned to innovate under the combined constraints of social, environmental, and competitive pressures, governments need new partners and operating models that support collaboration and innovation.

A climate of risk aversion is also limiting governments' capacity to innovate (Zussman, 2012; tinyurl.com/ 7dlwxm2). As result, governments are shifting some of their work into the community and private sectors. The federal government, for example, is reportedly exploring a range of new impact-investing tools, such as social impact bonds (socialfinance.ca/social-impact-bonds), as a means of financing innovation in social service delivery.

Yet another approach is to drive policy and program innovation through "labs" that draw on the specialized knowledge of multiple stakeholders, including affected populations, to develop and prototype new solutions. Denmark's Mind Lab (mind-lab.dk/en) and Finland's Design Lab (helsinkidesignlab.org) are two examples. Mindlab's Christian Bason, author of Leading Public Sector Innovation (2010; tinyurl.com/82r2nr8), revealed civil servants' untapped capacity to generate public service innovations during his recent talks in Canada (Bason, 2012; tinyurl.com/7eqeemw). Labs have a unique ability to reframe intractable problems, generate many potential approaches, winnow them down and rapidly test, adapt, and scale those with the highest potential for impact. Their premise is that, often, before we can succeed, we must fail several times. Rapid iteration helps sponsors to fail fast and safely, and it can generate superior outcomes, as Lisa Torjman's helpful blog post on the subject illustrates (Torjman, 2012; tinyurl.com/7zww9uy).

Another strategy is to release more government data to the public, on the premise that this generates transparency, ideas, and improved service standards. Montreal (tinyurl.com/7e6ndek) is the latest Canadian city to adopt this practice, joining Vancouver (tinyurl.com/873cpy9), Edmonton (data.edmonton.ca), Toronto (tinyurl.com/4rk9e4e), and Ottawa (tinyurl.com/2afd43z).

British Columbia is currently Canada's leading jurisdiction when it comes to public sector innovation. The BC Social Innovation Council (socialinnovationbc.ca) has produced several useful reports and the government is committed to implementing most of the Council's Action Plan. One example, BC Ideas (changemakers.com/ BCideas), uses a crowdsourcing platform to identify ideas and programs that "offer innovative solutions to health, social and environmental challenges facing BC communities today, and in the future."

\section{Social Innovation and the Community Sector}

There are 85,000 registered charities in Canada, employing two million people in fields such as education, health care, social services, arts and culture, environment, housing, and international development 


\section{Renewing the Future: Social Innovation Systems, Sector Shift, and Innoweave}

Stephen Huddart

(tinyurl.com/7glcx45). The sector's annual revenues of $\$ 177$ billion exceed those of the manufacturing and automotive sectors (Lasby, 2011; tinyurl.com/7syfm2m). Despite its size, the community sector does not have well-developed means for communicating its diversity and impact to itself, to Canadians, or their governments. Sub-sectors such as arts and culture and environmental organizations are often absent from sector councils, viewing themselves as worlds apart.

Charities are also finding that donors are demanding new levels of accountability and transparency, along with evidence of impact, rather than assurances of doing good. Absent agreed-upon standards for impact reporting however, it can be difficult for charities to assess or compare their performance.

Meanwhile, government's relationship with the sector has been coloured recently by maladroit actions that threaten to dampen free speech on matters of public interest (Globe and Mail, 2012; tinyurl.com/7twj2sc), as proposed new regulatory measures would require that charities report on all activities that can be construed as advocacy. Within the sector, reporting activities already constitute a significant drain on resources. A social services charity with an annual budget of less than $\$ 3$ million recounted to the author recently that it had to file 154 separate reports last year to funders -66 of them to governments - requiring the work of two full-time staff and $10 \%$ of its budget.

At a summit convened last November by Imagine Canada (imaginecanada.ca), the umbrella organization for the sector, participants from across Canada agreed on five key strategies for improving sector performance:

1. Recognize and promote charities and nonprofits as a sector.

2. Work collaboratively at the national and local levels for greater impact.

3. Identify and engage the next generation of leaders.

4. Leverage the diversity and ubiquity of the sector in dealings with business and government.

5. Employ technology to heighten interaction and effectiveness.

The full report of the summit can be read here: tinyurl.com/6lo4wrf
Amidst so much uncertainty, and with the additional pressure of having to accomplish as much or more with fewer resources, many community organizations are reluctant to risk new ways of working. At the same time, this is the sector that constitutes much of our capacity to create better futures, and it is in need of additional capacity to do so.

\section{Introducing Innoweave: Tools and Networks for Social Innovation}

Innoweave is currently in development by The J.W. McConnell Family Foundation (mcconnellfoundation.ca) and its partners in Social Innovation Generation (SiG; sigeneration.ca). As a social innovation system, it will employ a web platform, in-person workshops, experts, and consultants - as well as a repository of knowledge and skills SiG has amassed over the past five years - organized and presented to increase capacity for innovation in and with the community sector.

Innoweave modules will provide content on the tools and processes for implementing social innovations, including the nature of social innovation; systems mapping; impact and strategy formulation; social enterprise, social finance and impact investing; developmental evaluation; cloud computing; knowledge and community mobilization; change labs; and other topics.

At the core of each module, an Innoweave workshop will help organizations learn more about a particular tool and assess its applicability to their work. Basic workshop curricula will be available at no charge on the web platform, so that core components can be used by anyone. The site will also point to professional facilitators and authors of particular tools.

Workshop components will include:

1. A workshop agenda and facilitator's guide

\section{Presentation materials}

\section{Case studies}

4. Short videos that provide insight and examples from global experts, practitioners, and early adopters

5. Activities and exercises for board/management teams

6. Follow-up exercises designed to help users master the material. 


\section{Renewing the Future: Social Innovation Systems, Sector Shift, and Innoweave}

Stephen Huddart

While open access information and repeatable curriculum constitute an accessible starting point, many organizations will want to employ skilled facilitators and coaches to help them effectively implement new approaches. The web platform will provide information on scheduled in-person and web-based workshops, and it will list experts and consultants who can be called upon to provide assistance. Our goal is to also direct users to funding resources to which they can apply to support their use of professional consultants.

Peer learning and volunteer mentoring will also contribute to success. A community of practice linked to each module will facilitate exchange among organizations that are implementing a particular tool. Finally, the web platform will include links to other information hubs in Canada and around the world who share complementary goals. We envision co-producing or sharing modules with other such centres.

\section{Progress to Date and Next Steps}

A beta version of an Innoweave workshop, on cloud computing tools for community organizations (Platformation; platformation.ca), was organized in partnership with the Counselling Foundation of Canada (counselling.net) and Framework (frameworkorg.org) in November 2011. Over 90 people enrolled, and 16 of the organizations present have since launched cloud-computing projects aided by small grants from the Foundation. Each organization has a dedicated reporting page that it uses to share project milestones, expenses, and lessons learned with a community of other users. (The Foundation is reviewing another 40 applications submitted in connection with the project, and is not currently accepting more).

Discussions are underway with several prospective partners regarding a formal launch of Innoweave this fall.

\section{Conclusion: An Invitation to Collaborate}

Social innovation is coming of age. As sector boundaries shift and grow more porous, social innovation systems will enable us to collaborate more effectively and with greater impact, by putting complex problems at the centre of sustained efforts to transform them.
Recent advances in areas such as impact investing and technology-enabled platforms for scaling new ideas point to an approaching horizon where institutions and citizens can play a more active and informed role in shaping desirable and sustainable futures. The work will move forward more quickly when governments, companies, and civic organizations invest in active collaboration around solving complex social and environmental challenges.

In the rebalancing of sector responsibilities that occurs when governments downsize and companies work to advance blended value goals, we need to invest in the community sector's skills and professional capabilities around social innovation. Improving the sector's ability to adapt, to lead, and to participate in continuous social innovation will ultimately benefit us all.

\section{Acknowledgements}

I am grateful to Aaron Good, Tim Draimin, and Michael Lewkowitz for their invaluable contributions to earlier drafts of this paper.

\section{About the Author}

Stephen Huddart is the President and CEO of The J. W. McConnell Family Foundation in Montreal, where he has worked for the past nine years. The Foundation's mission is to engage Canadians in building a society that is inclusive, sustainable and resilient. Stephen's past endeavours include documentary filmmaking in Latin America, owning and operating a jazz cafe in Vancouver, and working as a humane educator with the British Columbia SPCA. He serves on the boards of ArtsSmarts, Philanthropic Foundations Canada, and the McGill Faculty of Religious Studies. He has a Masters Degree in Management from McGill University.

Citation: Huddart, S. 2012. Renewing the Future: Social Innovation Systems, Sector Shift, and Innoweave.

Technology Innovation Management Review. July 2012: (cc) BY 5-9. 